\title{
Correction to: Surveying the arable plant diversity of conventionally managed farmland: a comparison of methods
}

\author{
Alexander Wietzke • Christoph Leuschner
}

Published online: 16 June 2020

(C) Springer Nature Switzerland AG 2020

\section{Correction to: Environ Monit Assess (2020) 192: 98 https://doi.org/10.1007/s10661-019-8042-7}

The authors of the abovementioned publication noticed that the database for the beta diversity calculation was not correctly chosen. In addition, the description of the displayed beta diversity needed to be further specified for correct interpretation. The original calculation of beta diversity observed by the different survey methods was based on the Jaccard similarity index. However, the used function to calculate the differences in beta diversity of groups (function "betadisper" of the R package "vegan") is intended for the analysis of dissimilarity matrices instead of similarity matrices. The displayed beta diversity (Fig. 5) has to be read as the distance to the group spatial median based on the used beta diversity index (for detailed information, see function "betadisper").

The online version of the original article can be found at https://doi.org/10.1007/s10661-019-8042-7

A. Wietzke $(\bowtie) \cdot$ C. Leuschner

Department of Plant Ecology and Ecosystems Research, University of Goettingen, Untere Karspüle 2, 37073 Goettingen, Germany

e-mail: alexander.wietzke@biologie.uni-goettingen.de

C. Leuschner

e-mail: cleusch@gwdg.de
The authors apologize for the corrections that have become necessary (see below) and declare that these adaptions do not change the scientific conclusions drawn in any way.

The following corrections have been made:

- In the "Material and methods" section "Statistical analysis":

- The sentence "We also tested for differences between the survey methods in beta diversity among fields using Jaccard's similarity index as criterion (R package "vegan"; Oksanen et al. 2019)." should read as follows:

- "We also tested for differences between the survey methods in beta diversity among fields calculating the distances to group spatial medians based on Jaccard's dissimilarity index (R package "vegan," function "betadisper"; Oksanen et al. 2019)."

- In the "Results" section "Species composition and distribution":

○ The sentences "Beta diversity, measured with Jaccard's similarity index for the characteristic arable plant species, was higher for the Interior, Edge_30, and Edge_50 plot surveys (JI: 0.14- 
0.15; Fig. 5) than for the Edge_500, Corner, and Subplots surveys (JI: 0.17-0.22). Jaccard's index indicates lower floristic similarity among fields from the data retrieved with the former three methods." should read as follows:

- "Beta diversity, related to the characteristic arable plant species, was higher for the Interior, Edge_30, and Edge_50 plot surveys (medians 0.57-0.58; Fig. 5) than for the Edge_500, Corner, and Subplots surveys $(0.48-0.54)$. Calculated distances to the group spatial medians indicate lower floristic similarity among fields from the data retrieved with the former three methods."

- In the "Discussion" section:

$\circ$ The sentence "The effectiveness of this method is explained by the relatively low similarity in species composition in contemporary arable plant communities at the landscape scale, as is displayed in the low Jaccard indices and the relatively steep increase of the species-area curve at low plot numbers (Figs. 5 and 6)." should read as follows:

- "The effectiveness of this method is explained by the relatively low similarity in species composition in contemporary arable plant communities at the landscape scale, as is displayed by the quite high beta diversity and the relatively steep increase of the species-area curve at low plot numbers (Figs. 5 and 6)."

- The statistical results of pairwise comparisons with respect to beta diversity in the Electronic Supplementary Material 9 have been updated (no changes in significances).

- Figure 5 has been updated as follows (including the figure caption):

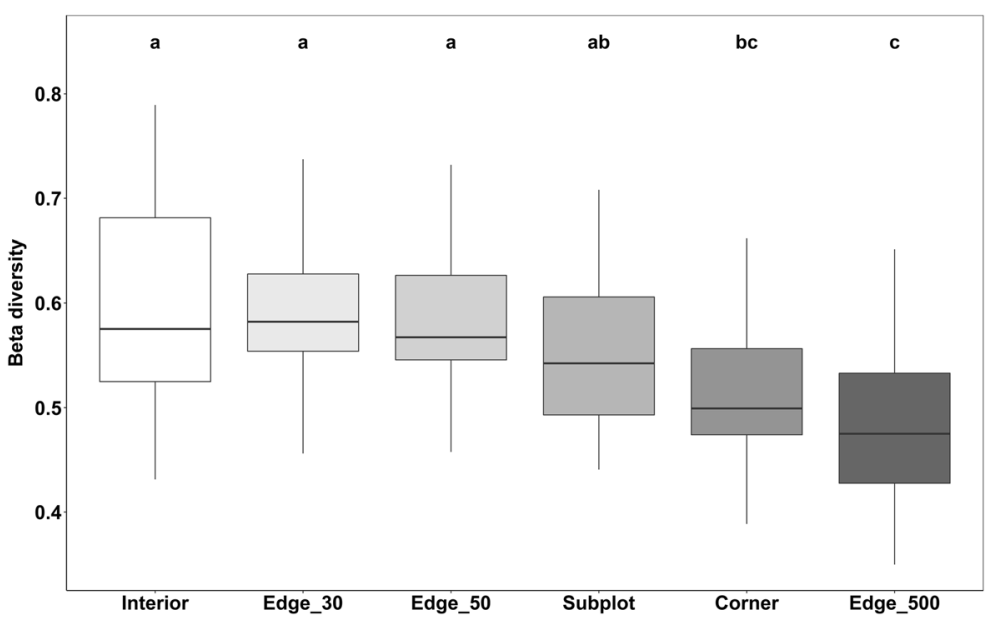

Fig. 5 Beta diversity, measured as distance to group spatial medians based on Jaccard dissimilarity index, for plots of the six survey methods in the 45 fields. Basis for the calculation were the number of arable plant species sensu stricto according to Hofmeister and Garve (2006). The lower the value, the more similar are species compositions for a given survey method (range of values between 0 and 1). $n=45$ for each survey method, except Interior $n=40$ (5 plots with no species were excluded); different small letters indicate significant differences between survey methods according to a Mann-Whitney U-Test $(\alpha \leq 0.05)$ 